\title{
Amortiguamiento Viscoso Equivalente en el Diseño Basado en Desplazamientos
}

\section{Equivalent Viscous Damping in Displacement Based Design}

\section{Cristian Bay}

Facultad Regional San Rafael - Universidad Tecnológica Nacional - Argentina cbay@frsr.utn.edu.ar

\section{Gustavo Palazzo}

Facultad Regional Mendoza - Universidad Tecnológica Nacional - Argentina gpalazzo@frm.utn.edu.ar

\section{Resumen}

A comienzos de siglo inicia el desarrollo de métodos de diseño basados en desempeño para estructuras sometidas a acción sísmica, destacándose entre ellos el método de diseño directo basado en desplazamientos (DDBD). Este método caracteriza el comportamiento no lineal de una estructura sometida a la acción sísmica mediante un sistema lineal equivalente, donde se utiliza el concepto de amortiguamiento viscoso equivalente (AVE) para representar el comportamiento no lineal, así como todas las fuentes de disipación de energía elásticas e inelásticas en la estructura. Los primeros desarrollos del AVE son de la década del 30 y con el advenimiento del DDBD se han realizado números estudios numéricos y experimentales para aproximar el comportamiento no lineal de la estructura. Este artículo presenta los principales avances del AVE en el DDBD. En las conclusiones se destaca la dependencia de varios factores y la necesidad de estudios experimentales.

Palabras claves: Amortiguamiento viscoso equivalente, Estado del arte, DDBD 


\begin{abstract}
At the beginning of the century the development of performance-based design methods for structures subject to seismic action starts, highlighting among them the direct design method based on displacements (DDBD). This method characterizes the non-linear behavior of a structure subjected to seismic action through an equivalent linear system, where the concept of equivalent viscous damping (AVE) is used to represent the non-linear behavior, as well as all sources of elastic energy dissipation. and inelastic in the structure. The first developments of the AVE are from the 30s and with the advent of DDBD, numerical and experimental studies have been carried out to approximate the non-linear behavior of the structure. This paper presents the main advances of the AVE in the DDBD. The conclusions highlight the dependence of several factors and the need for experimental studies.
\end{abstract}

Keywords: Equivalent Viscous Damping, State of Art, DDBD

\title{
Introducción
}

Los métodos de diseño basados en fuerzas se utilizan para definir la capacidad y la demanda de los sistemas estructurales sometidos a la acción sísmica. Sin embargo, es aceptado en la actualidad que las metodologías de diseño basadas en desplazamientos resultan más apropiadas, y han logrado superar las deficiencias de los tradicionales métodos basados en fuerzas (Priestley M., 1993). Una alternativa a los métodos basados en desplazamientos es el Diseño Directo Basado en Desplazamientos (DDBD) propuesto por Priestley, N. (1997). Mediante este enfoque, las estructuras se diseñan para alcanzar un objetivo de desempeño en vez de verificar los desplazamientos de un estado límite definido.

La estimación del desplazamiento máximo es un paso importante en el método DDBD, ya que, si los desplazamientos reales de la estructura son mayores que los desplazamientos considerados en el diseño, esta presentará demandas de ductilidad mayores a las esperadas y consecuentes aumentos en los niveles de daño o inestabilidad global del sistema. Para estimar este desplazamiento, el DDBD utiliza el concepto de amortiguamiento viscoso equivalente (AVE).

El AVE representa el comportamiento no lineal de la estructura mediante un sistema lineal equivalente, el cual es resuelto de forma simple y mediante expresiones de la dinámica clásica, evitando de esta forma complejos análisis dinámicos no lineales que requieren de tiempos computacionales extensos.

Las primeras investigaciones sobre AVE fueron desarrolladas por Jacobsen (1930) a principios del siglo pasado. Sus estudios fueron con señales armónicas y para una estructura de comportamiento bilineal. Luego diversos autores han desarrollado diversas investigaciones numéricas y experimentales para corroborar los procedimientos de Jacobsen frente a acciones dinámicas, y también para determinar nuevas relaciones que permitan estimar el desplazamiento máximo en el DDBD. (Dwairi et al., 2007)

Este trabajo tiene como objetivo recopilar los principales avances hasta la fecha en términos de AVE. Primero se presenta una revisión del DDBD, luego una referencia el concepto desarrollado por Jacobsen (1930) y después se abordan las principales investigaciones 
desarrolladas hasta la actualidad en términos de AVE en estructuras de edificios que son sometidas a la acción del sismo.

\section{Consideraciones generales del DDBD}

Tradicionalmente, la demanda sísmica se ha representado por un espectro de diseño en aceleraciones, evaluado para un 5\% de amortiguamiento. La relación entre la respuesta elástica e inelástica, en un importante rango de períodos que se aproximan a la zona donde se cumple el principio de igual desplazamientos (Veletsos et al., 1960), se utiliza para determinar la demanda en aceleraciones de la estructura inelástica. Esta demanda espectral puede ser aplicada en un análisis estático equivalente o modal espectral. A este método se lo denomina método de las fuerzas (Priestley et al., 2007).

A principios de este siglo comienzan a desarrollarse métodos de diseño basados en la respuesta máxima en desplazamientos en reemplazo de los métodos tradicionales basados en fuerzas. En general los métodos basados en desplazamientos realizan iteraciones para encontrar el desplazamiento máximo inelástico de la estructura, Sullivan et al. (2003) presenta un resumen de estos métodos y evalúa el desempeño de cada uno. Sin embargo, el DDBD presenta la particularidad que no requiere iteraciones en la determinación del desplazamiento máximo. Este método inicialmente fue desarrollado para puentes carreteros (Kowalsky, 1994, Priestley y Calvi, 1997) y posteriormente para edificios (Priestley et al., 2007).

El método DDBD utiliza el concepto de estructura sustituta, desarrollado por Shibata y Sozen (1976), en el cual la máxima respuesta del sistema no lineal de varios grados de libertad es representada por un sistema lineal viscoelástico con determinadas propiedades de rigidez y amortiguamiento equivalente. Conceptualmente, el procedimiento de la estructura sustituta se descompone en dos etapas, la representación de un sistema de varios grados de libertad como un sistema de un grado de libertad, por un lado, y la aproximación de la no linealidad de la estructura (material y geométrica) por propiedades lineales equivalentes por el otro. A continuación, se resumen los pasos del procedimiento del DDBD (Priestley y Calvi, 1997).

a.- Determinación de la demanda sísmica: La demanda sísmica en el DDBD se determina en base a un espectro de diseño en desplazamientos para un amortiguamiento del 5\% en un sistema de un grado de libertad (Figura 1).

b.- Selección de un desplazamiento objetivo, $\Delta_{\text {sys }}$ : El desplazamiento objetivo es determi nado de acuerdo con criterios de desempeño, como puede ser distorsión de piso o deformación máxima del acero, entre otros. Se transforma la estructura de múltiples grados de libertad en un sistema de un grado de libertad mediante el concepto de estructura sustituta (Shibata y Sozen, 1976).

c.- Determinación del desplazamiento de fluencia, $\Delta_{\mathrm{y}}$ : El desplazamiento de fluencia del sistema equivalente se determina mediante iteraciones en el diseño.

d.- Determinación del AVE, $\xi_{\text {eq }}$ : los valores de AVE se analizan en el presente trabajo y se obtienen en función del ciclo de histéresis de la estructura y de la ductilidad de desplazamiento, $\mu$, obtenida como el cociente entre el $\Delta_{\text {sys }}$ y el $\Delta_{\mathrm{y}}$ estimado. En algunos casos interviene el período de la estructura y el factor de rigidez posterior a la fluencia. Con el AVE se reduce el espectro de desplazamientos elásticos, mediante el factor $\eta$, hay diversas expresiones en la bibliografía, aunque la expresión (1) es la recomendada en el DDBD para sismos de campo lejano. (Priestley et al., 2007 
e.- Determinación del período efectivo, $\mathrm{T}_{\mathrm{ef}}$, y el corte basal, $\mathrm{V}_{\mathrm{B}}$ : $\mathrm{El} \mathrm{T}_{\mathrm{ef}}$ se obtiene en el espectro de desplazamientos (Figura 1), al ingresar con el $\Delta_{\text {sys }}$ e interceptar la curva del AVE definido en el punto anterior. Con las expresiones de la dinámica clásica para sistemas de un grado de libertad se puede obtener la rigidez efectiva, $\mathrm{K}_{\mathrm{ef}}$, $\mathrm{y}$ el corte basal, expresiones (2) y (3).

f.- Análisis estructural y diseño seccional: El corte basal obtenido de la expresión (3) es distribuido como fuerzas laterales equivalentes. Sobre la base de los resultados de un análisis elástico se determinan las fuerzas de demanda de los elementos estructurales.

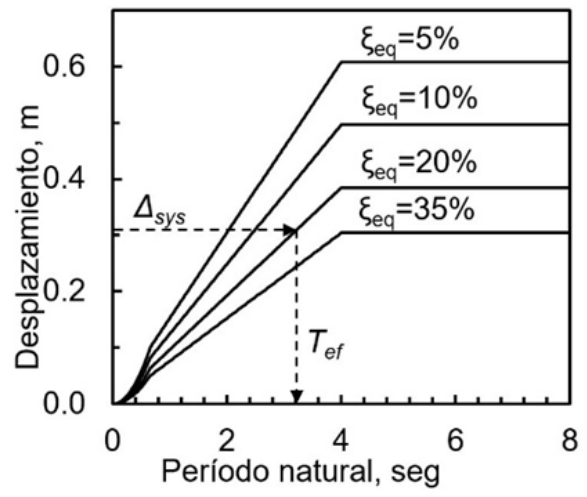

Figura 1. Espectro de respuesta en desplazamientos.

$$
\begin{aligned}
& \eta=\left(\frac{0.07}{0.02+\xi}\right)^{0.5} \\
& K_{e f}=\frac{4 \pi^{2} M}{T_{e f}^{2}} \\
& V_{B}=K_{e f} \Delta_{s y s}
\end{aligned}
$$

\section{Concepto inicial del AVE}

Para comprender como el amortiguamiento viscoso equivalente se ha desarrollado en el tiempo, es necesario remontarse a principios del siglo XX. Jacobsen (1930) propone por primera vez representar la respuesta de un amortiguador viscoso no lineal mediante un sistema lineal equivalente. El mismo autor, luego aplica el mismo concepto a sistemas histeréticos no lineales (Jacobsen, 1960). Las hipótesis en que se basa el análisis son: ambos sistemas presentan el mismo período inicial y no se considera cambios en el período, ambos sistemas se encuentran sometidos a una acción armónica de amplitud constante, ambos sistemas se encuentran en resonancia y la energía disipada en cada ciclo del sistema no lineal es igual a la energía disipada en el sistema equivalente lineal. 
Estas hipótesis no se cumplen cuando el sistema es sometido a una acción sísmica, la respuesta no es armónica y el desplazamiento máximo ocurre antes de que la respuesta transitoria se amortigüe. Por ello, se esperan diferencias importantes entre los desplazamientos reales y los obtenidos mediante el método propuesto por Jacobsen, especialmente en el rango de períodos cortos en el cual se presenta mayor dependencia del período fundamental del sistema.

Desde sus comienzos, el AVE se evalúa como la suma del amortiguamiento elástico, $\xi_{v}$, y el amortiguamiento histerético, $\xi_{\mathrm{h}}$, expresión (4), el cual representa la disipación por el comportamiento no lineal de la estructura.

El amortiguamiento elástico se utiliza para representar diversos mecanismos como fisuración en el hormigón armado, la no linealidad en la fase elástica de la respuesta, la interacción con elementos no estructurales y la interacción suelo-estructura, entre otros. En general se considera entre el $2 \%$ y el $5 \%$ del amortiguamiento crítico y no es objeto del presente trabajo.

El amortiguamiento histerético considera el amortiguamiento viscoso basado en el concepto de energía disipada $\left(\mathrm{E}_{\mathrm{D}}\right)$ y energía elástica $\left(\mathrm{E}_{\mathrm{E}}\right)$. Al igualar la energía disipada de un amortiguador viscoso con la energía disipada del comportamiento no lineal histerético, resulta el factor amortiguamiento histerético mostrado en la expresión (5). Si ambos sistemas se encuentran bajo excitación armónica y la frecuencia de excitación, $\omega$, es la misma que la frecuencia natural, $\omega_{n}$, del sistema de un grado de libertad se obtiene a la expresión (6). Donde $\mathrm{A}_{\mathrm{h}}$ representa el área dentro del ciclo, $\mathrm{F}_{0}$ la fuerza máxima y $\mathrm{U}_{0}$ el desplazamiento máximo (Jacobsen, 1960). En la Figura 2 se presenta el ciclo de histéresis para un sistema viscoso y para un sistema histerético.

Por lo desarrollado en los párrafos anteriores es clave que el AVE resulta un factor determinante en la metodología del DDBD, ya que permite estimar el comportamiento no lineal de la estructura, evitando trabajar con sistemas no lineales complejos de resolver.

Diversas expresiones han sido desarrolladas en la bibliografía para determinar el AVE. En la sección siguiente se desarrolla un resumen de los trabajos desarrollados a la fecha.

$$
\xi_{e q}=\xi_{v}+\xi_{h}
$$
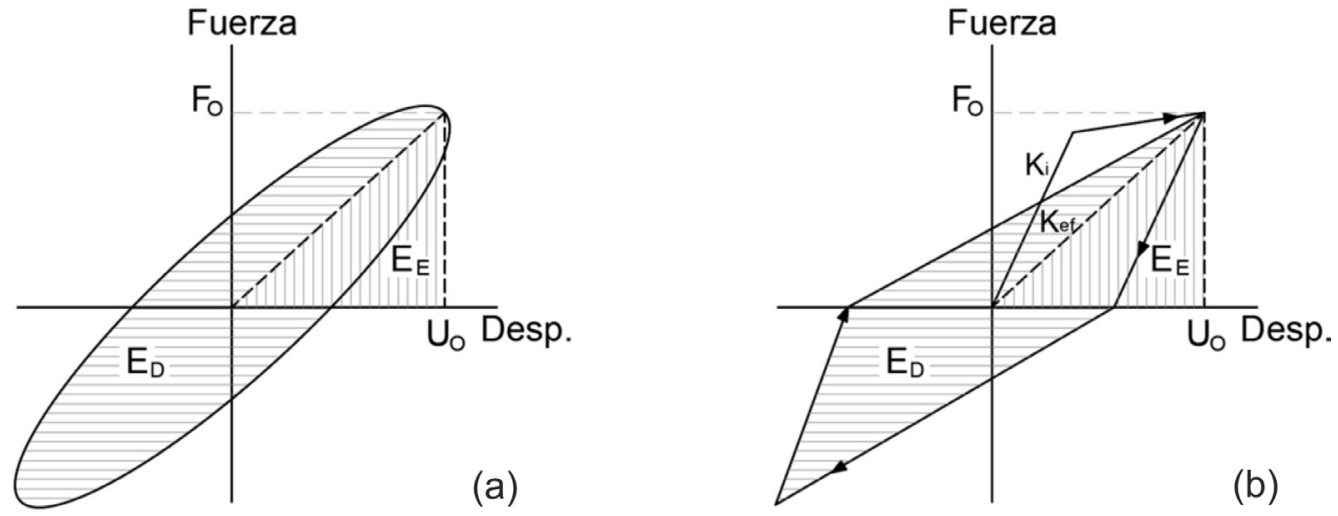

Figura 2. Fuerzas disipativas y elásticas para (a) Amortiguamiento viscoso y (b) Amortiguamiento histerético. 


\section{Avances del AVE}

Como se mencionó anteriormente Jacobsen $(1930 ; 1960)$ fue quien propone el primer procedimiento aplicando el concepto de AVE. A continuación, se presentan los principales desarrollos propuestos por otros investigadores para estimar el AVE y se realizan gráficos para comparar las expresiones.

Rosenblueth y Herrera (1964) modifican el enfoque propuesto inicialmente por Jacobsen (1960), al igualar la energía disipada en un ciclo de histéresis armónico con la misma cantidad para un sistema viscoelástico lineal en resonancia. Al sistema equivalente lineal se le asigna una rigidez efectiva, $\mathrm{K}_{\mathrm{ef}}$, obtenida como la rigidez secante al desplazamiento máximo de la respuesta. Aplicando la expresión (6) al sistema visco-elástico mostrado en la Figura $2 \mathrm{~b}$ se obtiene la expresión (8) para estimar el AVE. También, de la Figura $2 \mathrm{~b}$ se puede deducir la relación entre el período inicial, $\mathrm{T}_{\mathrm{i}}$, $\mathrm{y}$ el período efectivo, Tef, expresión (7). Este enfoque se ha utilizado en distintos métodos de diseño de estructuras sometidas a acción sísmica. (Shibata y Sozen, 1976).

En la Figura 3 se presenta la variación del AVE en función de la ductilidad para distintos valores de rigidez posterior a la fluencia, r. Se observa cómo se reduce el AVE conforme aumenta el valor de $\mathrm{r}$. También se presenta la relación entre el periodo efectivo y período inicial, en este caso la relación disminuye conforme se incrementa el valor de $\mathrm{r}$.

$$
\begin{aligned}
\frac{T_{e f}}{T_{i}} & =\sqrt{\frac{\mu}{r \mu-r+1}} \\
\xi_{e q} & =\xi_{v}+\frac{2}{\pi} \frac{(\mu-1)(1-r)}{\mu[1+r(\mu-r)]}
\end{aligned}
$$

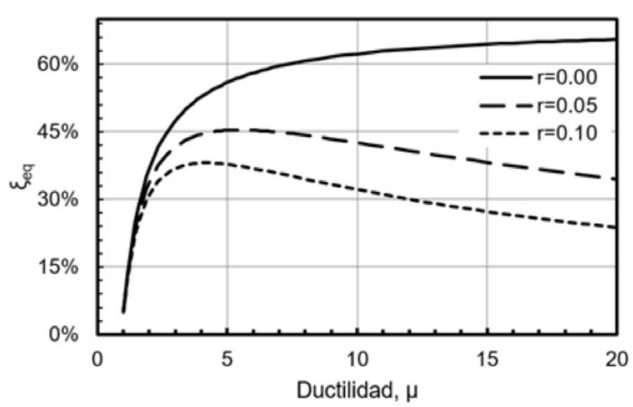

(a). Amortiguamiento histerético, $\xi_{\text {eq. }}$

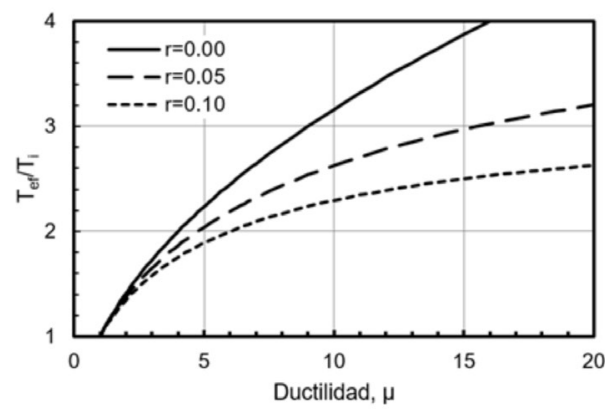

(b) Relación de periodos efectivo e inicial, $T_{e} / T$

Figura 3. Expresiones (7) y (8) propuestas por Jacobsen (1960).

Gulkan y Sozen (1974) proponen relacionar el período efectivo con la rigidez secante en el desplazamiento máximo. El AVE se determina igualando la energía de la excitación del sistema y la energía disipada por un amortiguador ficticio equivalente, expresión (9), donde $\beta_{\mathrm{s}}$ es el amortiguamiento equivalente, $\mathrm{t}_{\mathrm{f}}$ es la duración de la excitación, $\ddot{y}$ es la aceleración de la excitación y la velocidad relativa de la estructura de un grado de libertad. Mediante 
resultados experimentales, y aplicando el modelo de histéresis desarrollado por Takeda et al. (1970), se obtiene la expresión (10) para determinar el AVE en columnas de hormigón armado. Este modelo no presenta dependencia del factor r. En la Figura 4 se presenta la expresión (10).

$$
\begin{aligned}
& \beta_{s}\left(2 m \omega_{s} \int_{0}^{t_{f}} \dot{x}^{2} d t\right)=-m \int_{0}^{t_{f}} \ddot{y} \dot{x} d t \\
& \xi_{e q}=\xi_{v}+0.2\left(1-\frac{1}{\sqrt{\mu}}\right)
\end{aligned}
$$

Iwan y Gates (1979) aplican un enfoque estadístico para determinar el AVE y el período efectivo óptimo. Consideran 12 registros sísmicos y seis modelos de histéresis en el estudio. La optimización consiste en minimizar el error medio cuadrático de la diferencia entre desplazamientos del sistema no lineal y el sistema lineal equivalente. La expresión (11) representa el modelo promediando la energía y el amortiguamiento en cada ciclo de histéresis. En la Figura 4 se presenta la expresión (11).

$$
\xi_{e q}=\xi_{v}+\frac{6(1-r)(\mu-1)^{2}+\pi \xi_{v}\left[(1-r)\left(3 \mu^{2}-1\right)+2 r \mu\right]}{2 \pi \mu^{2}[1+r(\mu-1)+(1-r) \ln \mu]}
$$

Posteriormente, Iwan (1980) propone una expresión que estima la variación entre el período inicial y el período secante y la expresión (12) para determinar el AVE para un modelo histerético, derivado de una combinación del sistema lineal y sistema de deslizamiento de Coulomb. La variación del AVE se muestra en la Figura 4.

$$
\xi_{e q}=\xi_{v}+0.0587(\mu-1)^{0.371}
$$

Kowalsky (1994) utiliza la variación en el período basado en la rigidez secante al máximo desplazamiento en la respuesta y el enfoque propuesto por Jacobsen (1930) para desarrollar una relación de amortiguamiento equivalente dado por la expresión (13). El sistema se basa en el modelo de histéresis propuesto por Takeda (1970) con un factor de descarga de 0,50 y rigidez posterior a la fluencia igual a cero. La variación es mostrada en la Figura 4.

$$
\xi_{e q}=\xi_{v}+\frac{1}{\pi}\left(1-\frac{1}{\sqrt{\mu}}\right)
$$


Kwan et al. (2003), aplican el mismo enfoque estadístico sugerido por Iwan y Gates (1979) y proponen relaciones empíricas entre el período efectivo y el período inicial de seis ciclos de histéresis. Analizan estructuras con períodos entre 0,1 y 1,5 segundos y sometidas a 20 registros sísmicos. La expresión (14), Figura 4, permite estimar el AVE para el caso de sistemas elastoplásticos de baja y alta degradación, característicos de las estructuras dúctiles de acero y hormigón armado. No se considera el endurecimiento post fluencia del material en este estudio.

$$
\xi_{e q}=0.352 \mu \xi_{v}+\frac{0.717}{\pi} \frac{\mu-1}{\mu}
$$

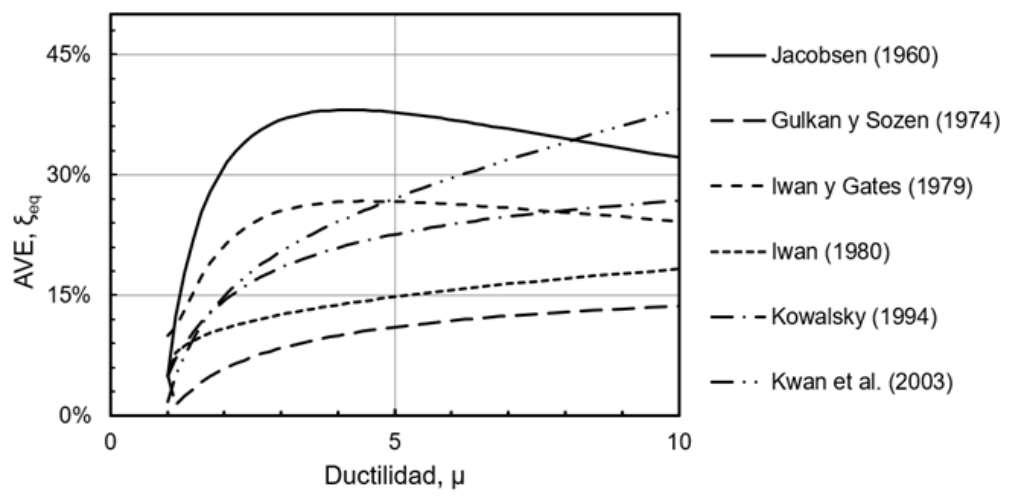

Figura 4. $A V E, \xi_{\text {eq }}$ propuestas por distintos autores.

Blandon y Priestley (2005) realizan un estudio sobre el AVE. Con el fin de cumplir un amplio rango de sistemas de disipación y utilizan seis ciclos de histéresis: (i) Takeda Largo (TL), con $\alpha=0.50$ y $\beta=0.00$, utilizado para pilas de puentes y muros de hormigón armado, (ii) Takeda Corto (TC), con $\alpha=0.30$ y $\beta=0.60$, para representar el comportamiento de pórticos de hormigón armado, (iii) Bilinear (Otani, 1981) para simular estructuras aisladas con péndulo de fricción con $\mathrm{r}=0.20$, (iv) Elastoplástico (Otani, 1981) para representar el comportamiento de estructuras con aisladores de goma, (v) Ramber Osgood (Otani, 1981) apropiado para simular estructuras metálicas y (vi) Ring Spring generalmente utilizado para estructuras pretensadas de hormigón armado con juntas hibridas. En los ciclos de Takeda a es el factor de descarga de rigidez y $\beta$ el factor de recarga de rigidez (Takeda et al., 1970).

Utilizan seis acelerogramas, uno sintético ajustado a la norma europea (EC 8-I, 2005) y cinco artificiales escalados al espectro de diseño ATC32 para suelo tipo C, con PGA = 0,7 g (ATC-32, 1996) . Aplican cinco niveles de ductilidad (2 a 6) y 8 períodos efectivos entre 0,5 seg y 4 seg para la modelación.

En la primera parte del trabajo se comparan los resultados obtenidos con los propuestos por Jacobsen, al igual que estudios anteriores se arriba a una sobre estimación del AVE, sobre todo en el caso de ciclos de histéresis con gran absorción de energía. 
En la segunda parte se proponen expresiones para determinar el AVE. La expresión (15) presenta la forma general obtenida basada en estudios de Priestley (1993).

$$
\begin{gathered}
\xi_{e q}=\xi_{v}+\frac{a}{\pi}\left(1-\frac{1}{\mu^{b}}-0.1 r \mu\right)\left(1+\frac{1}{(T+c)^{d}}\right) \frac{1}{N} \\
N=1+\frac{1}{(0.5+c)^{d}}
\end{gathered}
$$

Donde a, b, c y d son constantes para cada ciclo de histéresis, $\mathrm{N}$ el factor de normalización (16), y los demás parámetros han sido definidos anteriormente. Una diferencia importante con las expresiones deducidas por otros autores es la dependencia del período en el AVE. La Tabla 1 muestra los valores de las constantes para los distintos ciclos de histéresis y la Figura 5 presenta la variación de AVE en función de la ductilidad, para $\mathrm{T}_{\mathrm{ef}}=1$ segundo. Se puede observar la sobreestimación del AVE de Jacobsen (1960).

Blandon y Priestley (2005) obtienen dispersiones del 10\% para ductilidades entre 3 y 6, cuando la ductilidad es inferior la dispersión aumenta hasta el 20\%. Además, indican que en el caso de sistemas bilineales se deberían realizar estudios con otros valores de factor de endurecimiento post fluencia. Cabe destacar que la dependencia del $\mathrm{T}_{\mathrm{ef}}$ en la determinación del AVE hace que el procedimiento sea iterativo.

\begin{tabular}{|c|c|c|c|c|}
\hline \multirow{2}{*}{ Ciclo de histéresis } & \multicolumn{4}{|c|}{ Constantes } \\
\cline { 2 - 5 } & $\mathrm{a}$ & $\mathrm{b}$ & $\mathrm{c}$ & $\mathrm{d}$ \\
\hline Takeda Largo, TL & 130 & 0.50 & 0.85 & 4 \\
\hline Takeda Corto, TC & 095 & 0.50 & 0.85 & 4 \\
\hline Elasto Plástico, EEP & 140 & 0.50 & 0.85 & 2 \\
\hline Bilineal, BL & 160 & 0.50 & 0.85 & 4 \\
\hline Ramberg Osgood, RO & 150 & 0.45 & 1.00 & 4 \\
\hline Ring Spring, RS & 050 & 0.50 & 1.00 & 3 \\
\hline
\end{tabular}

Tabla 1. Coeficientes para el AVE propuestos por Blandon y Priestley (2005) 


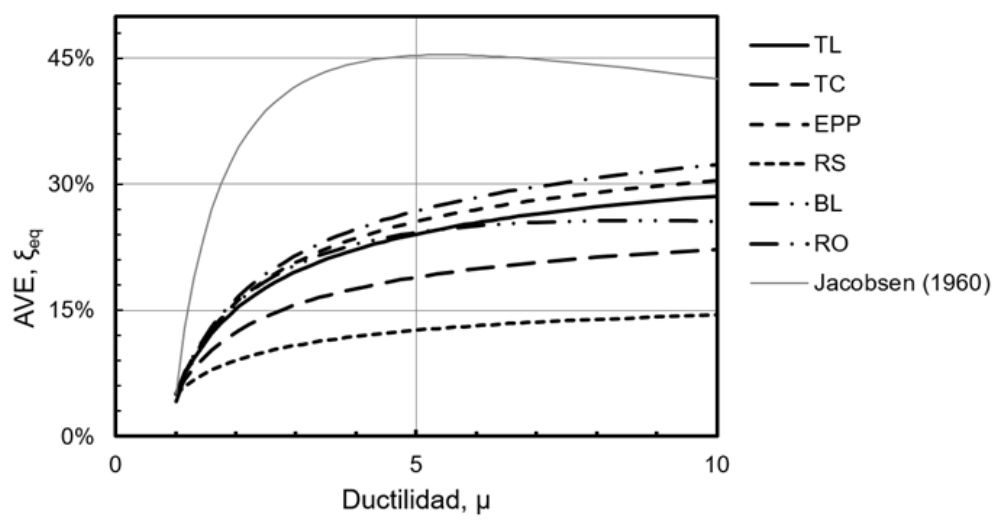

Figura 5. AVE, $\xi_{\text {eq' }}$ propuesto por Blandon y Priestley (2005) para $T_{\text {ef }}=1$ segundo.

Dwairi et al. (2007) realiza un estudio con dos propósitos: (1) identificar la dispersión que hay entre el AVE propuesto por Jacobsen combinado con la rigidez secante utilizada en el DDBD, y el amortiguamiento obtenido de un análisis tiempo historia no lineal; y (2) establecer coeficientes de ajuste entre ambos amortiguamientos para ser aplicados al DDBD. El estudio se realiza para cuatro ciclos de histéresis distintos: (i) elastoplástico (EPP) característico de estructuras de acero, (ii) Takeda corto (TC), con $\alpha=0.5$ y $\beta=0.0$, característico de columnas y tabiques de hormigón armado, (iii) Takeda largo (TL), con $\alpha=0.0$ y $\beta=0.6$ referente a vigas de hormigón dúctil y (iv) Ring-Spring característico de estructuras postensadas y con sistemas de aislamiento sísmico. Utiliza una base de 100 registros sísmicos y ductilidades entre 1 y 6.

$\mathrm{Al}$ igual que estudios anteriores, se corrobora la sobreestimación del amortiguamiento para períodos superiores a 1 segundo. Además, para períodos inferiores a 0,40 segundos se observa una subestimación del AVE.

En base al estudio numérico realizado se proponen nuevas expresiones para considerar el AVE en función de la ductilidad, el período efectivo y la forma del ciclo de histéresis. Se utiliza la expresión (17), que presenta una forma similar a la propuesta por Jacobsen.

$$
\xi_{e q}=\xi_{v}+C \frac{\mu-1}{\pi \mu}
$$

Donde C depende del ciclo de histéresis y del período efectivo (Tabla 2). La relación entre los desplazamientos del análisis dinámico no lineal y el análisis lineal equivalente se encuentran cercano a la unidad en todos los casos, excepto para el elásto-plastico, donde la relación supera a la unidad. La dispersión de los valores, para los 100 registros, resulta entre $20 \%$ y $40 \%$ en general.

En la Figura 6 se presenta la gráfica para periodos mayores a 1 segundo. Estos valores resultan significativamente menores que los obtenidos por Jacobsen. 


\begin{tabular}{|c|c|c|}
\hline \multirow{2}{*}{ Ciclo de histéresis } & \multicolumn{2}{|c|}{ Coeficiente C } \\
\cline { 2 - 3 } & $\mathrm{T}_{\text {eff }} \leq 1 \mathrm{seg}$ & $\mathrm{T}_{\text {eff }}>1 \mathrm{seg}$ \\
\hline Takeda Largo, TL & $65+50\left(1-T_{\text {ef }}\right)$ & 65 \\
\hline Takeda Corto, TC & $50+40\left(1-T_{\text {ef }}\right)$ & 50 \\
\hline Elasto Plástico, EPP & $85+60\left(1-T_{\text {ef }}\right)$ & 85 \\
\hline Ring-Spring, RS & $30+35\left(1-T_{\text {ef }}\right)$ & 30 \\
\hline
\end{tabular}

Tabla 2. Coeficientes para la expresión de AVE propuestos por Dwairi (2007)

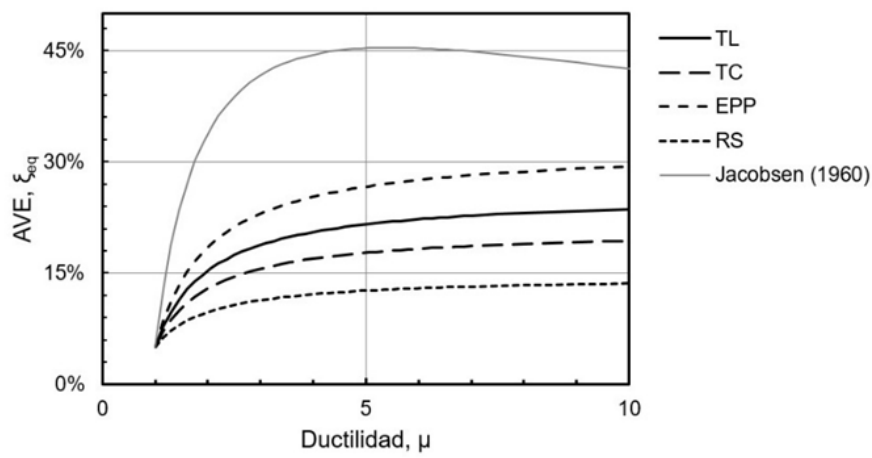

Figura 6 .AVE, $\xi_{\text {eq' }}$ propuesto por Dwairi et al. (2007), para $T_{\text {ef }}>1$ segundo.

Ghaffarzadeh et al. (2014) desarrollan expresiones para estimar el AVE de pórticos de hormigón armado con diagonales dúctiles de acero. El estudio se basa en modelaciones numéricas de pórticos de 4, 6, 8, 12, 16 y 20 pisos, sometidos a registros escalados de alta sismicidad. Se obtiene la expresión (18), de forma similar a las desarrolladas por Blandon y Priestley (2005). Los autores también realizan ensayos a escala reducida de pórticos de hormigón armado con diagonales de acero para validar la expresión propuesta.

$$
\xi_{e q}=\xi_{v}+\frac{70}{\pi}\left(1-\frac{43}{\mu^{4}}-4.7 \times 10^{-5} \mu^{4}\right)
$$

Tao Liu desarrolla investigaciones del AVE en sistemas histeréticos bilineales con diferentes relaciones de endurecimiento post fluencia. Liu et al. (2014a) comparan catorce expresiones de la bibliografía para sistemas con aislamiento de base. Considera períodos iniciales entre 0,1 segundo y 1,50 segundos, ductilidades entre 2 y 50 sometidos a una base de 12 registros sísmicos con magnitud entre 6.1 y 7.5 y PGA superior a $0.10 \mathrm{~g}$. Observan que las expresiones analizadas obtienen buena estimación del AVE solo en ciertos rangos de períodos o para determinadas ductilidades, incluso para algunas relaciones de endurecimiento post fluencia. Pero no logran una expresión general para sistemas bilineales que permita una estimación adecuada del AVE en función del período, ductilidad y factor de endurecimiento 
post fluencia.

Liu et al. (2014b) continúan con la investigación anterior y, partiendo de la expresión de Jacobsen(1930), desarrollan la expresión (19) para determinar el AVE en función de la ductilidad, período inicial y factor de endurecimiento post fluencia.

$$
\xi_{e q}=\xi_{v}+\frac{2(1-r)(\mu-1)}{\pi \mu[1+r(\mu-1)]} \times \frac{1}{\left(0.7763+0.2886 T_{i}\right)+\frac{\left(0.5651+1.8410 T_{i}\right)}{\exp (r \mu)}}
$$

Con esta expresión general para sistemas bilineales se obtiene buena aproximación del AVE en estructuras con períodos iniciales entre 0,1 segundo y 1,5 segundos, ductilidades entre 2 y 50 y relaciones de endurecimiento post fluencia entre 0,02 y 0,20. En la Figura 7 se presenta la curva de AVE propuesta por Liu et al. (2014b) y se contrasta con la de otros autores. Se observa que los niveles de amortiguamiento obtenidos son inferiores a los obtenidos por Blandon y Priestley (2005) para el mismo factor de endurecimiento post fluencia.

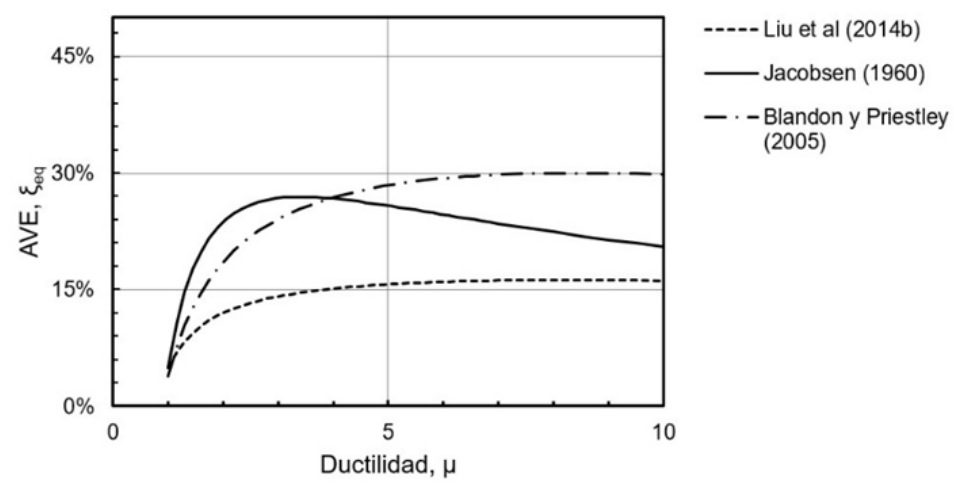

Figura 7 . Relaciones de amortiguamiento para sistemas bilineales con $\mathrm{Ti}=1$ segundo.

En los puntos anteriores se realizó una revisión de la literatura sobre el AVE aplicado al DDBD. Se destaca una importante cantidad de trabajos numéricos en contraste con la falta de estudios experimentales.

También se resalta que las expresiones dependientes del ciclo de histéresis presentan menor dispersión en la determinación del AVE.

Otro parámetro clave es el periodo efectivo, Blandon y Priestley (2005), lo consideran en sus expresiones y obtienen resultados con menor dispersión que los de Dwairi et al. (2007). Aunque resulta en un proceso iterativo debido a que el periodo efectivo no es un parámetro conocido al inicio del DDBD.

Para estructuras de hormigón armado se recomienda la utilización de las expresiones de Dwairi et al (2007) que presentan una cantidad importante de validaciones numéricas y no dependen del período. En el caso de estructuras con sistemas de protección sísmica, caracterizadas por ciclos de histéresis bilineal, se recomienda utilizar la expresión desarrollada por Liu et al. (2014b) dado que depende del factor de endurecimiento posterior a la fluencia. 


\section{Conclusiones}

Este trabajo presenta una revisión del avance del conocimiento en el amortiguamiento viscoso equivalente (AVE) como factor de linealización en el diseño directo basado en desplazamientos (DDBD).

Las expresiones disponibles en la bibliografía permiten cubrir una importante cantidad de sistemas estructurales considerando como parámetros principales la forma del ciclo de histéresis, la ductilidad de desplazamientos del sistema y en algunos casos el periodo efectivo y el factor de endurecimiento posterior a la fluencia.

Para estructuras de hormigón armado se recomiendan las expresiones que solo dependen de la ductilidad de desplazamiento y para estructuras con sistemas de protección, aislamiento sísmico o disipación de energía, se recomiendan expresiones que dependan del factor de endurecimiento posterior a la fluencia y el periodo efectivo.

Por último, remarcar que resulta necesario realizar estudios experimentales para contrastar los resultados numéricos disponibles en la literatura. 


\section{Referencias}

ATC-32. (1996). "Improved Seismic Design Criteria for California Bridges: Provisional Recommendations". California: Applied Technology Council.

Blandon, C., y Priestley, M. (2005). "Equivalente viscous damping equations for direct displacement based design". Journal of Earthquake Engineering, 9(2), 259-278.

Dwairi, H., Kowalsky, J., y Nau, J. (2007). "Equivalent Damping in Support of Direct Displacement-Based Design". Journal of Earthquake Engineering, 11(4), 512-530.

EC 8-I. (2005). "Structure is seismic regions - Design. Part 1, General and Building". Belgica: Commission of European Communities.

Ghaffarzadeh, H., Jafari, A., y Zafari, N. (2014). "Equivalent viscous damping in direct displacement-based design of steel braced reinforced concrete frames". Struct. Design Tall Spec. Build., 23, 604-618.

Gulkan, P., y Sozen, M. (1974). "Inelastic response of reinforced concrete structures to earthquake motion". ACI Journal, 71, 604-610.

Iwan, W. (1980). "Estimating inelastic spectra from elastic spectra". Earthquake Engineering and Structural Dynamics, 8, 375-388.

Iwan, W., y Gates, N. (1979). "Estimating earthquake response of simple hysteretic structures". Journal of Engineering Mechanics Division, ASCE, 105, 391-405.

Jacobsen, L. (1930). "Steady forced vibratios as influenced by damping". ASME Transactione, 52,169-181.

Jacobsen, L. (1960). "Damping in composite structures". Proceedings of the Second World Conference on Earthquake Engineering, (págs. 1029-1044). Tokio, Japan.

Kowalsky, M. (1994). "Displacement-based design-a methodology for seismic design applied to RC bridge columns". University of California at San Diego, La Jolla, California.: Master's Thesis.

Kwan, W., y Billington, S. (2003). "Influence of hysteretic behavior on equivalent period and damping of structural systems". Journal of Structural Engineering, ASCE, 129(5), 576-585.

Liu, T., Zordan, T., Briseghella, B., y Zhang, Q. (2014a). "Evaluation of equivalent linearization analysis methods for seismically isolated buildings characterized by SDOF systems". Engineering Structures, 59, 619-634.

Liu, T., Zordan, T., Briseghella, B., y Zhang, Q. (2014b). "An improved equivalent linear model of seismic isolation system with bilinear behavior". Engineering Structures, 61, 113126.

Otani, S. (1981). "Hysteresis models of reinforced concrete for earthquake response analysis". Journal of Faculty of Engineering, University of Tokyo, 36(2), 407-441.

Priestley, M. (1993). "Myths and fallacies in earthquake engineering - conflicts between design and reality". NZ National Society for Earthquake Engineering, (3),1-26.

Priestley, M., y Calvi, G. (1997). "Concepts and procedures for direct displacement-based design". Seismic Design Methodologies for the Next Generation of Codes, 171-181. 
Priestley, M., Kowalsky, M., y Calvi, M. (2007). "Displacement Based Seismic Design Of Structure". Pavia, Italia, Pavia: IUSS Press.

Rosenblueth, E., y Herrera, I. (1964). "On a kind of hysteretic damping". Journal of the Engineering Mechanics Division, ASCE, 90(4), 37-48.

Shibata, A., y Sozen, M. (1976). "Substitute structure method for seismic design in R/C". Journal of the Structural Division, ASCE, 102(1), 1-18.

Sullivan, T., Calvi, G., Priestley, M., y Kowalsky, M. (2003). "The limitations and performances of different displacement based design methods". Journal of Earthquake Engineering, 7(1), 201-241.

Takeda, T., Sozen, M., y Nielsen, N. (1970). "Reinforced concrete response to simulated earthquakes". Journal of the Structural Division ASCE, 96(12), 2557-2573.

Veletsos, A., y Newmark, N. (1960). "Effect of inelastic behavior on the response of simple systems to earthquake motions". Proceedings of the 2nd World Conference on Earthquake Engineering, (págs. 2,895-912). Tokyo, Japan. 